Rev. salud pública. 12 sup (2): 67-70, 2010

Poster Presentation

\title{
Mycobacteria of veterinary interest
}

\section{Production and potency of PPDs Mycobacterium phlei and Mycobacterium fortuitum isolated soils from La Pampa-Argentina}

Amelia Bernardelli ${ }^{1}$, Bernardo Alonso ${ }^{2}$, Delia Oriani ${ }^{3}$

1 SENASA, Dirección de Laboratorio y Control Técnico(DILAB),Lab. de Referencia en Paratuberculosis y Tuberculosis Bovina de la OIE, Buenos Aires-Republica Argentina.

2 SENASA (DILAB).

3 Universidad Nacional de La Pampa, Facultad de Ciencias Veterinarias, Cátedra de Microbiología, Gral. Pico, La Pampa -Republica Argentina.

The Non-Tuberculous Mycobacteria (NTM), whose habitat the environment has acquired importance in the last years because of the immunosupressed patients, infected HIV ,and also in develop countries that have managed to eradicated the bovine tuberculosis. Where it has been verified that certain NTM interfere in the diagnosis of tuberculosis when it is applied to the delayed hypersensitivity test with purified protein derivative (PPD) tuberculin from Mycobacterium bovis. In the works to field exists controversy about the relevance of these environmental mycobacteria when control and eradication of animal tuberculosis are applied.The production of PPDs from the isolated soils from the province of La Pampa and the verification of the possible crossed reaction with bovine tuberculin PPD, prescribed test for international trade.

Two lots of PPDs corresponding of $M$. phlei and $M$. fortuitum were elaborated with a protein content of $1.5 \mathrm{mg} / \mathrm{mL}$ both.Guinea pigs were sensitized with dead $M$. phlei, $M$. fortuitum and $M$. bovis.After 60 days the potency tests were made bioassay at the guinea pigs, using also like standard of reference bovine PPD,Lot.NÂ०5 DILAB and employing a Latin square design.

The relative activity in sensitized with $M$. bovis guinea pigs was of $2.18 \%$ with the $M$. phlei PPD and $0.4 \%$ with $M$. fortuitum PPD.According to the observed it has been demonstrated that the environmental NTM does not present a relevant action in the cell-mediated response test in guinea pigs.

Key Words: Non Tuberculous Mycobacteria (NTM), Purified Protein Derivative (PPD), Crossed reactions.

\section{Diagnosis of tuberculosis in bovine herds with low prevalence of the disease in the Argentina republic}

Sergio Garbaccio ${ }^{1}$, Fernando Delgado $^{1}$, Liliana Rodríguez $^{1}$, Pablo Huertas $^{1}$, Martín Zumarraga $^{2}$, AliciaAlito', Agustín Venzano', Winston Morris ${ }^{1}$, Carlos Garro $^{1}$, Daniel Funes ${ }^{1}$

1 Instituto de Patobiología e

2 Instituto de Biotecnología - CICVyA, INTA Castelar, Buenos Aires. Argentina.

Bovine tuberculosis (TB) is a worldwide zoonosis. Mycobacterial infection is diagnosed by intradermal reaction (IDR) in Argentine cattle herds. In many herds preva- 
lence of TB is low (under $1 \%$ ) whereas other ones become positive after IDR, showing a minimal number of reactors.

The aim of this study was to explore the actual health status in low TBC prevalence herds through alternative diagnostic tests. Nasal swabs as well as milk, blood, and tissue samples were taken from IDR-positive animals belonging to ten cattle herds where TB prevalence was under $1 \%$. Bacteriology, histopathology, molecular biology (PCR) and immunology [gamma interferon (IFN- $\gamma$ )] were the methods performed. We considered a herd as positive when one isolation of Mycobacterium bovis was achieved. Mycobacterium bovis was isolated in nine out of ten herds. Lesions consistent with the disease and a positive PCR were observed in at least one animal out of these herds. IFN- $\gamma$ was coincident with IDR in $70 \%$ of the cases. The remaining herd did not result positive to any of the performed tests.Nine out of the ten herds were considered positive. The results were consistent with IDR in $90 \%$ of the cases except for one herd where IDR appeared non-associated with the other tests performed. This point remains to be investigated yet. Thus, we consider relevant to set new complementary diagnostic tests in herds where both the presence of the etiologic agent or the existence of false positive reactors needs to be confirmed.

Key Words: Bovine tuberculosis, zoonosis, false-positive.

\section{Identification of potential antigens in Mycobacterium avium subsp. paratuberculosis cell wall}

Gabriela Echeverría Valencia ${ }^{1}$, Wendy Xolalpa ${ }^{2}$, Ignacio Etchechoury ${ }^{1}$, Andrea Gioffre $^{1}$, Guillermo Mendoza Hernandez ${ }^{3}$, María Isabel Romano ${ }^{1}$, Clara Espitia ${ }^{2}$

1 Instituto de Biotecnología, CICVyA, INTA. Castelar, Provincia de Buenos Aires. Argentina.

2 Departamento de Inmunología, Instituto de Investigaciones Biomédicas, Universidad Nacional Autónoma de México. México, D.F., México.

3 Departamento de Bioquímica, Facultad de Medicina, Universidad Nacional Autónoma de México. México, D.F., México.

Mycobacterium avium subsp. paratuberculosis (MAP) is the causative agent of bovine paratuberculosis a significant economical disease in cattle.

Identification of potential proteins candidates for serodiagnosis and vaccine development is one of the most important research objectives in bovine paratuberculosis. The mycobaterial cell wall is a subcellular fraction known to play a major role in pathogenesis. This fraction contains antigenic proteins, which are involved in cellpathogen interaction, and molecules which play a role in attachment to mononuclear phagocytes. The aim of this investigation was to recognize and identify potential antigens from MAP's cell wall. In order to achieve our purpose, we obtained MAP cell wall proteins. The cell wall extract was separated and resolved by $2 \mathrm{D}$ electrophoresis and then incubated with bovine sera from paratuberculosis-infected animals. Previously, each serum was adsorpted with Mycobacterium phlei extract to increase specificity of reactions. The reactive spots were selected based on reactivity, pl and mass. The identification of proteins was deduced by N-terminal and/or MS. In this work we identify potencial antigens from Mycobacterium aviun subsp paratuberculosis. 
Key Words: Antigens, Paratuberculosis, Cell Wall.

\section{Mycobacterium species monitoring in wild and domestic animals from Colombia}

Claudia Castro ${ }^{1}$, Claudia Brieva ${ }^{2}$, Karol Barragán $^{2}$, Diego Soler $^{2}$, Iván Rubiano ${ }^{3}$, y Wellman Ribón ${ }^{1}$

1 Grupo de Micobacterias, Instituto Nacional de Salud.

2 Facultad de Medicina Veterinaria y de Zootecnia, Universidad Nacional de Colombia.

3 Fundación Zoológico Colombiano. Bogotá D.C., Colombia.

INTRODUCTION: Mycobacterium species can infect man and other vertebrates including reptiles, birds and mammals, and cause tuberculosis and mycobacteriosis characterized by a generalized dissemination of the infection, varying clinical signs and visible pathological changes which affect individuals as well as animal populations. Direct contact between animals and humans may contribute to disease transmission both ways. OBJECTIVE: To monitor the presence of mycobacteria in wild animals kept captive in fauna centers, captured when in free-living conditions or in domestic animals. METHODS: Between July 2005 and March 2008, blood samples, gastric aspirates, oropharyngeal swabs and internal organs of 124 mammals (9 genres), five birds (2 genres) and a reptile were studied. Samples were processed for microbiological analysis through cultures in Löwenstein Jensen medium and DNA extraction for molecular detection by amplifying and analysing the $h s p 65$ gene restriction pattern. RESULTS: In two mammals gendres four $M$. fortuitum isolates were identified through culture and molecular analysis; there was no second sample, nor compatible clinical signs so infection was discarded. In birds, an M. gadium isolate was identified through molecular methods and later confirmed by the necropsy and the histopatological analysis. No M. tuberculosis complex species were detected in the samples under study. CONCLUSIONS: Surveillance of mycobacteria in free-living and captured wild animals, as well as in domestic animals, is important given the growing contact with humans that may be immunocompromised, and also to preserve individuals and animal populations health.

Key Words: Mycobacteriosis, immunocompromised, domestic animals, free-living.

\section{Diagnosis of Mycobacteria important for veterinary medicine in Colombia}

Rafael Villalobos A. Ivonne Hernández T. Víctor Tibata R. Esperanza Rueda de Clavijo

Instituto Colombiano Agropecuario - ICA, Laboratorio Nacional de Diagnóstico Veterinario (LNDV).

The Colombian Agricultural Institute, ICA, is the official entity responsible for prevention, control and erradication of bovine tuberculosis in Colombia. The National Laboratory of Veterinary Diagnosis (LNDV) of the Institute meets conditions of infrastructure and competent human resources to offer diagnosis of the disease by bacteriological, pathological, molecular and serological methodologies. The Mycobacterium bovis was isolated for the first time in the country in 1972 from a bull imported from Europe, 
but since 1961 there was histopathological evidence. Since its creation, the ICA has performed monitoring of cattle in slaughter plants and the identification and subsequent slaughter of positive reactors to the tuberculin skin test. In order to optimize the classic diagnosis of this zoonotic disease (Ziehl Neelsen colouring, biological tests in experimental animals, pathology and bacterial isolation), in the last decade ICA has implemented new methodologies such as a polymerase chain reaction (PCR), reported in 1995 (Rodriguez et al) as a specie-specific technique. To support field diagnosis LNDV offers capture ELISA technique for the detection of cattle specific gamma interferon. Due to the presence of mycobacteria avium complex in cattle and sheep, ICA also performs diagnostic techniques in paratuberculosis.

Key Words: Veterinary Diagnosis, eradication, zoonotic, paratuberculosis. Mycobacterium bovis. 